\title{
RELEVANCE OF THE SPEED-AGILITY REACTION IN THE PREPARATION OF RUGBY SEVENS PLAYERS
}

\author{
Maria-Emilia VIZITIU ${ }^{1 *}$, Viorel COJOCARU ${ }^{1}$, Dan BADEA ${ }^{1}$ \\ ${ }^{1}$ National University of Physical Education and Sport, Faculty of Physical Education and Sport, \\ Bucharest, Romania \\ *Corresponding author: vizitiu_emilia@yahoo.com
}

https://doi.org/10.35189/dpeskj.2020.59.s.7

\begin{abstract}
The success of female rugby sevens players is largely based on their high motor potential as well as the individual and collective tactical skills. Most training programmes specific to the game of rugby sevens emphasise the development of travel speed, unwillingly neglecting the progress of the reaction speed (in specific training) and the agility, which are found in the technical and tactical actions of the game. The purpose of this research was to assess the efficiency of both specific training programmes and female players. The research was conducted throughout a training macrocycle in the first months of the competitive year 2019. The research subjects were the female players in the junior team of the "Agronomia" Sports Club of Bucharest and some players from the National Olympic Youth Centre. The research methods used are: scientific documentation, observation, measurement, statistical and mathematical method, graphical method. The experimental approach to the issue was highlighted by processing and interpreting the results obtained after applying the proposed tests. Therefore, the priority in the training programmes specific to the game of rugby sevens is to maintain and improve the execution of movements from the perspective of speed as a motor skill performed with an optimal manifestation of agility, all these aspects being reflected in the efficiency of technical and tactical actions.
\end{abstract}

Keywords: female rugby sevens, agility, reaction speed.

\section{Introduction}

Speed is a complex action of the musculoskeletal system when solving a task that requires a quick response from the athlete. The two characteristics of speed are that it can be similar to the movement pattern, which means that it can be cyclic and acyclic.

The factors that can affect speed in sport are:

- Biochemical processes;

- Muscles;

- Brain;

- Kinematics;

- Interaction between neural and muscular systems;

- Genetics.

According to Duthie et al. (2003), all players in rugby union are required to compete and maintain ball possession along with performing their specific roles in the forward and back positions; these physical demands and game concepts make a fundamental difference between rugby union and other rugby codes such as rugby sevens.

"Professionalism in rugby union has placed emphasis on the strength and conditioning field as a key component for success. Strength and conditioning staff formulate physical development programs based on the specificity of rugby union, thus focusing on the critical 
components of performance to optimize outcomes. However, there is little available evidence regarding the role of objective performance testing in rugby union.” (Green, 2011, p. 1256)

Small yet important differences in patterns of play and contrasting anthropometric characteristics of players in rugby union and league codes exist (Gabbett et al., 2009); "thus, it would not be advisable to assume that field test protocols developed for rugby league could be directly applied to rugby union. Therefore, there is a need to develop reliable field test protocols for rugby union to measure key skills and responses to strength and conditioning interventions. To do this, field tests must be designed toward replicating the demand components of the game." (Green, 2011, p. 1257)

There are also a series of muscular factors that can influence speed, namely:

- Fibre type;

- Pennation angle;

- Fibre viscosity;

- Connective tissue;

- Muscle elasticity;

- Fascicle length;

- Intramuscular coordination;

- Co-contraction;

- Intermuscular coordination;

- Basic strength;

- Maximum strength;

- Power;

- Explosive power;

- Muscle relaxation.

According to Docherty et al. (1988), the ability to change direction both quickly and accurately, as needed, is considered by many to be an integral part of athletic performance. The ability to move quickly helps athletes perform better, increasing their movement accuracy and movement speed (Abernethy, 1993). Broadly, this is often referred to as "agility".

Galpin et al. (2008) state that, although agility is essential to performance, few data exist regarding the accuracy and reliability of agility testing methodology and even fewer data regarding the effectiveness of training programmes designed to improve agility performance. The majority of these studies measure change-of-direction (COD) tasks where the individual is not forced to make decisions or react.

Agility and change-of-direction abilities in high-level sports have been extensively studied. Authors such as Lloyd and Oliver (2012) claim that a top priority in training sessions should be represented by these attributes, especially during childhood and adolescence. Basically, general motor skills and qualities connected with the change of direction and agility may be developed during the periods mentioned above (Van Hooren \& De Ste Croix, 2020). If we consider the physical and mental changes that are related to these periods of development towards maturity, the reliability of change-of-direction and agility performance may differ during objective testing (Taylor et al., 2019). Changes in height, body mass and sprint speed are highlighted in this period of maturation, which leads to large differences in results when performing change-of-direction and agility tasks. As a general point of view, 
maturity status and absolute strength are related to performance and the ability to absorb and improve strength throughout maturation (Pichardo et al., 2019). The onset of "adolescence awkwardness" around peak height velocity seems to increase biological variance in test performance in adolescent athletes (Taylor et al., 2019).

Researchers such as Gabbett (2005) classify rugby as an activity that requires body contact and is played in many countries around the world. Rugby players take part in multiple tackles as well as short-duration and high-intensity running efforts. They need to have improved fitness and the right skills to meet all the psychological and physiological demands while performing a variety of offensive and defensive tasks during the game, especially the ability to make the best motor decision under pressure and fatigue.

Most people who play this sport would characterise agility as any body movement that involves quick changes of direction. Sheppard (2006) says that agility can be defined as a rapid, whole-body change of direction or speed in response to a sport-specific stimulus. Several specialists were interested in assessing pre-planned COD speed tests, while others, such as Farrow et al. (2005), have lately begun to study the perceptual components of agility. In this regard, the ability of team players to anticipate a specific game action stimulus was tested. Sheppard (2006) demonstrated that a reactive agility test applied to a team of Australian football players had much better results than a pre-planned COD test. Researchers such as Gabbett (2006) and Farrow et al. (2005) showed similar results for netball and softball players. Moreover, a significant correlation $(r=0.74)$ was reported between linear speed and COD speed performance, the connection between reactive agility and pre-planned COD speed having the tendency to be moderate in magnitude. The findings of Young et al. (2002) suggest that agility performance is limited by physical and perceptual factors.

Fast physical activities also include rugby sevens, energy-based activity being the key element to game play. Even if this game seems to be violent, it is one of the most interesting and enjoyable contact sports. As a valid proof worldwide, the game of rugby sevens has not only grown in popularity due to its spectacular character, but also the level at which the tournaments organized by the World Rugby Series and Rugby Europe has exceeded by far the imagination of lovers of this sport.

Rugby is practised under several forms, and each of them is defined by a motor quality or a relationship of more than one to dominate the development of the game. In the case of rugby sevens, the game differs in terms of speed and its forms of manifestation, building a close connection between them and the game-specific motor skills.

In the opinion of Cârstea (2000), motor qualities are attributes of the human body, which can be developed or educated. Ontogenetically, people are born with some indices of these qualities that develop naturally since birth until a certain age, and then begin to decline at different rates determined by a multitude of variables.

Ursanu (2015) states that speed is one of the most important qualities available to the human body and is part of the category of conditional skills, also being a spatial-temporal characteristic of movement.

According to Marcus (2006), rugby sevens is the most intense game version, which is full of fast offensive and defensive actions from both sides of the field, and this is an opportunity for players to perform as many technical actions as possible in a short period of time. 
Authors like Peterson et al. (2006) tried to explain the role of other performance markers, such as sprint speed and leg extensor power, on agility performance. Other researchers (Cressey et al., 2007) assessed the effect of chronic training on COD performance by infusing a variety of training modalities, such as COD drills, unstable surface training, general strength training and jump squat training, into the training programme of current athletes.

Rugby sevens is another version of the classic game of rugby but also a contact sport played in many countries around the world. The game is physically demanding, requiring players to take part in many body contacts and submaximal effort sequences, such as shortduration actions or distances covered at maximum intensity. Players must have the highest level of physical preparation and skills to perform a wide range of offensive and defensive actions needed in competitions.

In the opinion of international authors and coaches, agility is the word that defines the ability to act and change the direction and position of the body quickly and efficiently in due time. It is thought to be a key component of specific physical training in rugby sevens, which is why special attention and an effective preparation plan are needed.

Studies conducted by specialists in the field, such as Gabberth (2008), tend to assess the variation of two aspects represented by speed and change of direction in rugby players, but the interesting fact is that none of them has tried to assess their reactive agility.

Sheppard et al. (2006) state that agility requires speed in changing direction but also perceptual and decision-making skills and reaction speed, an opinion with which we completely agree, these being important aspects in playing this dynamic game.

\section{Problem statement}

Favourite players for rugby sevens are the fastest and sharpest ones, who can express the technique of the game at the highest level. The difference from the classic game of rugby is that players must have excellent peripheral vision and be aware of the created space.

Regardless of our role in the world of rugby, we can always obtain the best performance only if we are willing to focus on the opportunities offered by each game separately, each version of the game of rugby involving problems related to understanding and transmitting it.

To be able to assess the motor level of rugby sevens players, we need to identify their potential through physical and technical tests. According to the game of rugby sevens, physical testing is based on the assessment of motor skills and abilities in speed conditions.

Rugby sevens is a speed and energy game that is practiced by the sharpest players. The quality of the game is influenced by the players' physical performance, the area where they operate and the environmental conditions in which they control their activity. Speed, strength, explosive power, reaction speed and execution speed are the main determinants of physical performance, and therefore applying a scientific method is essential for their improvement.

The ability to play rugby with width and speed is the key to successful attack. In rugby sevens, players should aim to occupy the full width of the pitch, effectively positioning themselves in seven imaginary lanes equally spread across the field (Marcus, 2006). 


\section{Purpose of study}

The study aims to demonstrate the efficiency of an optimal training programme for improving short-distance running speed, reaction speed and execution speed in female rugby sevens players through appropriate testing.

Along with the goals of this research, we used the necessary studies for applying the experiment, namely specific reaction and execution speed exercises and speed running adapted to the technical and tactical content.

An important role was played by the materials with which the sports activity was conducted, helping the players to appreciate and be aware of their efficiency.

According to the results recorded at the beginning of the training programme, the female rugby sevens players from the "Agronomia" Sports Club of Bucharest followed a speed development programme, with an emphasis on their reaction speed to acoustic and visual stimuli and their execution speed in correlation with technical and tactical actions. We present below the physical tests, followed by an overview of the training and development programme.

- 10-m speed run: aims to assess the linear starting and acceleration speed. Running is performed in a straight line as fast as possible; the player starts running at the coach's signal from a standing position simultaneously with the start of the timer; as soon as the player has crossed the finish line, the timer will be stopped.

- 5 x 10 m shuttle run: the player runs over a 5-m distance and returns to the starting line in the same way, this back-and-forth movement being performed 10 times.

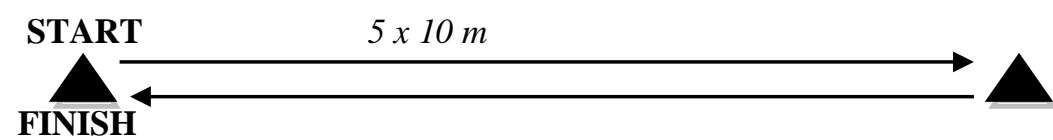

- Illinois test: aims to assess the execution speed (agility), the ability to travel with a rapid change of direction from different angles. The surface on which the movement is performed has $10 \mathrm{~m}$ and $5 \mathrm{~m}$, respectively, and the distance between the cones is $3.3 \mathrm{~m}$. The player must run following the route shown in the image below.

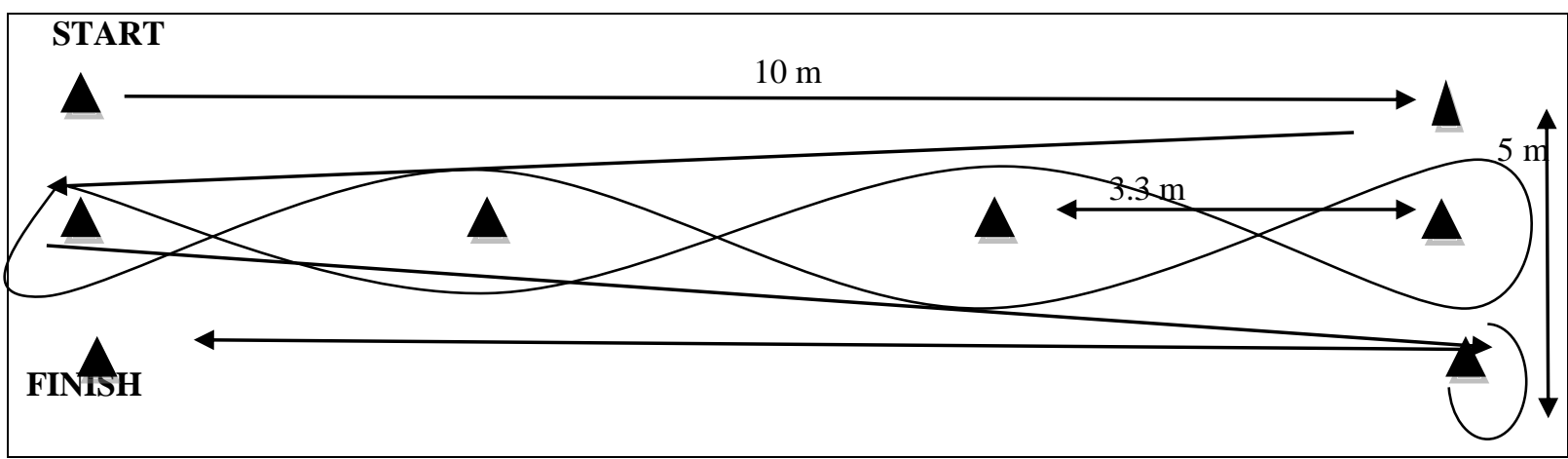


- Ball reception from high-start running: aims to assess the reaction speed to visual stimuli. Players are in a high-start position and move by running at speed with the intention of catching the ball. The distance between the starting line and the ball is 2 $\mathrm{m}$ wide, and the ball is in the hands of the coach, whose arms are outstretched forward at a hight of $1.50 \mathrm{~cm}$. To complete the test, five balls are used for each player, and the number of balls received is recorded.

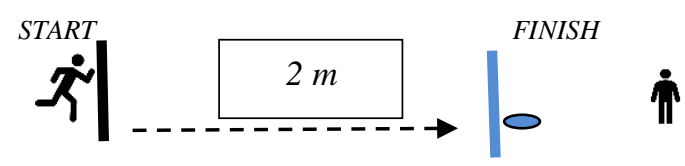

- Passing speed while running: the player starts running and receives a pass from a teammate located on the left side at a 5-m distance and immediately passes the ball to the right-side teammate, goes around the cone positioned $5 \mathrm{~m}$ ahead and returns to the starting line executing the same actions (i.e. reception and passing). The route must be covered three times consecutively to receive and pass with precision before reaching the cone. Passes executed beyond the cone or those without precision will not be taken into account.

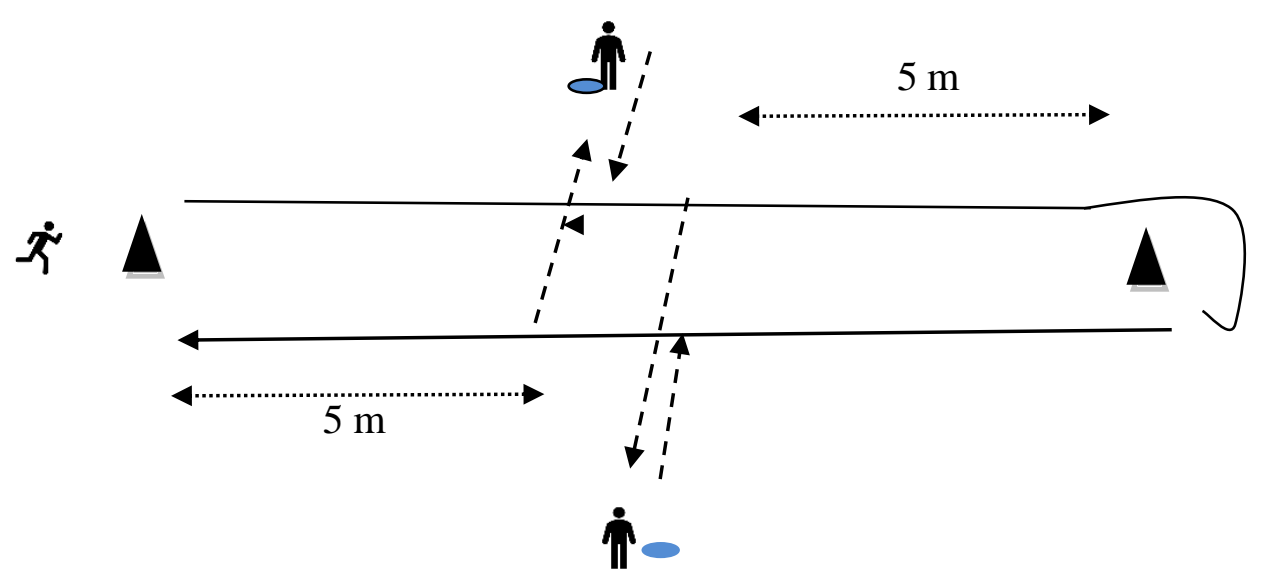

Ground pass speed: the player has to alternately pass six balls to two teammates positioned at $5 \mathrm{~m}$ on the left and right sides. The execution must be accurate and within 15 seconds. Exceeding this timeframe will affect the number of passes executed, approximately one pass per second. 


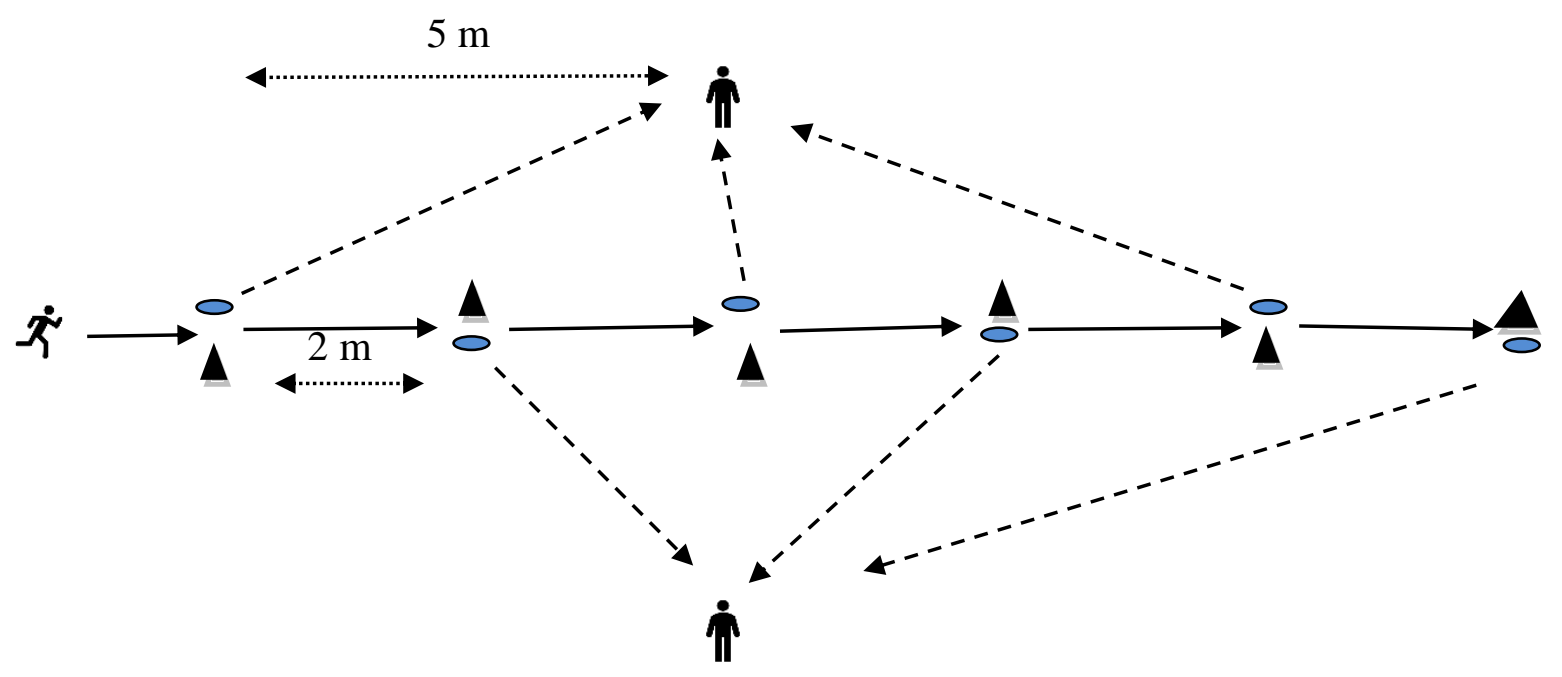

\section{Methodology}

For the current study, we used an experimental design to compare the results recorded after applying the tests in the two different periods of time. Participants in this study were 14 players aged between 16 and 18 years, who followed the training programme over a 4-month period, from January to April, in the competitive year 2019. We have to mention that all players are at the beginner level, with a rugby experience between 1 and 4 years. The testing was divided into three categories and was applied two days apart as follows:

- 10-m Speed run, 5 x 10 m shuttle run

- Ball reception, Illinois test

- Ball reception, running pass and ground pass

\section{Results}

After 14 weeks of training, the tests revealed a significant improvement in the reaction time and execution time. Simultaneously, an improvement in the execution time of motor skills was observed. Table 1 shows the results obtained from the 6 tests, before and after the end of the training programme, highlighting the times that indicate the motor potential of rugby players.

Table 1. Initial and final assessments for the six tests applied

\begin{tabular}{ccc}
\hline Test & Initial test & Final test \\
\hline 10 m SPEED RUN & $2 " 97-3 " 88$ & $2 " 68-3 " 1$ \\
5 X 10 m SHUTTLE RUN & $18 " 68-21 " 60$ & $16 " 66-20 " 51$ \\
ILLINOIS TEST & $15 " 92-18 ” 02$ & $15 " 40-17 " 81$ \\
BALL RECEPTION & $1-3$ & $2-5$ \\
RUNNING PASS & $2-5$ & $4-6$ \\
GROUND PASS & $3-5$ & $4-6$ \\
\hline
\end{tabular}


Based on the test batteries, the graphical representation below shows the number of training sessions that the two categories of female players needed depending on their experience on the sports field. As expected, players with more experience on the playing field needed a shorter period to consolidate their skills in contrast to the others. To fulfil this main purpose, it was established a 3-week period, namely 9 training sessions dedicated to the consolidation period. Figures 1 and 2 show the number of training sessions for the specific motor preparation of rugby sevens players.

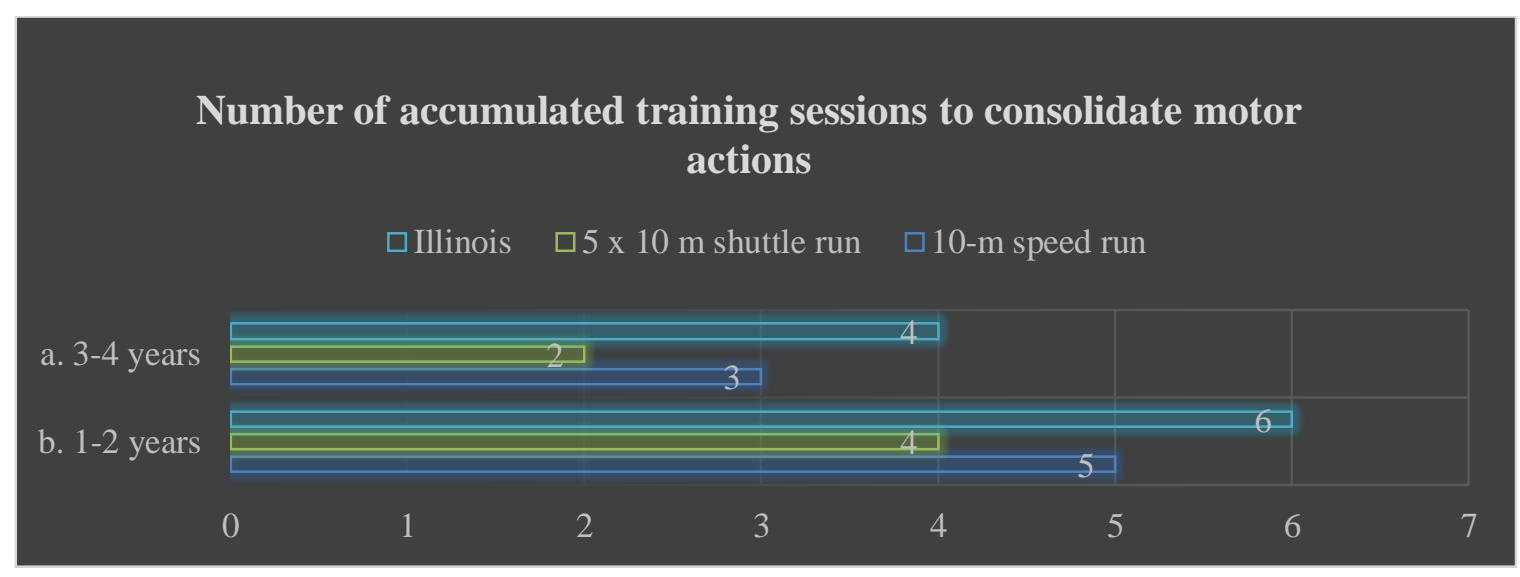

Figure 1. a. Number of training sessions for 3-4 years of experience; b. Number of training sessions for 1-2 years of experience

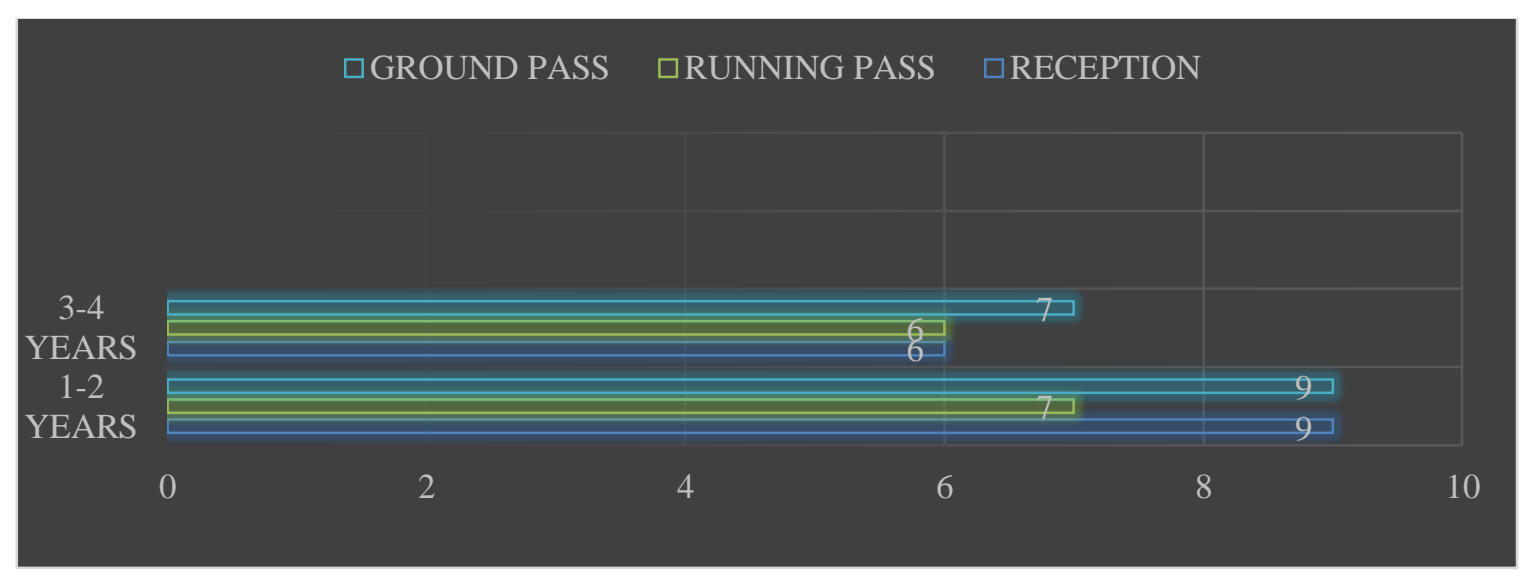

Figure 2. Number of accumulated training sessions to consolidate motor skills

Figures 3 and 4 show the difference in motor potential for the tested group in terms of physical quality of motor skills, specifying the best and worst times recorded. 


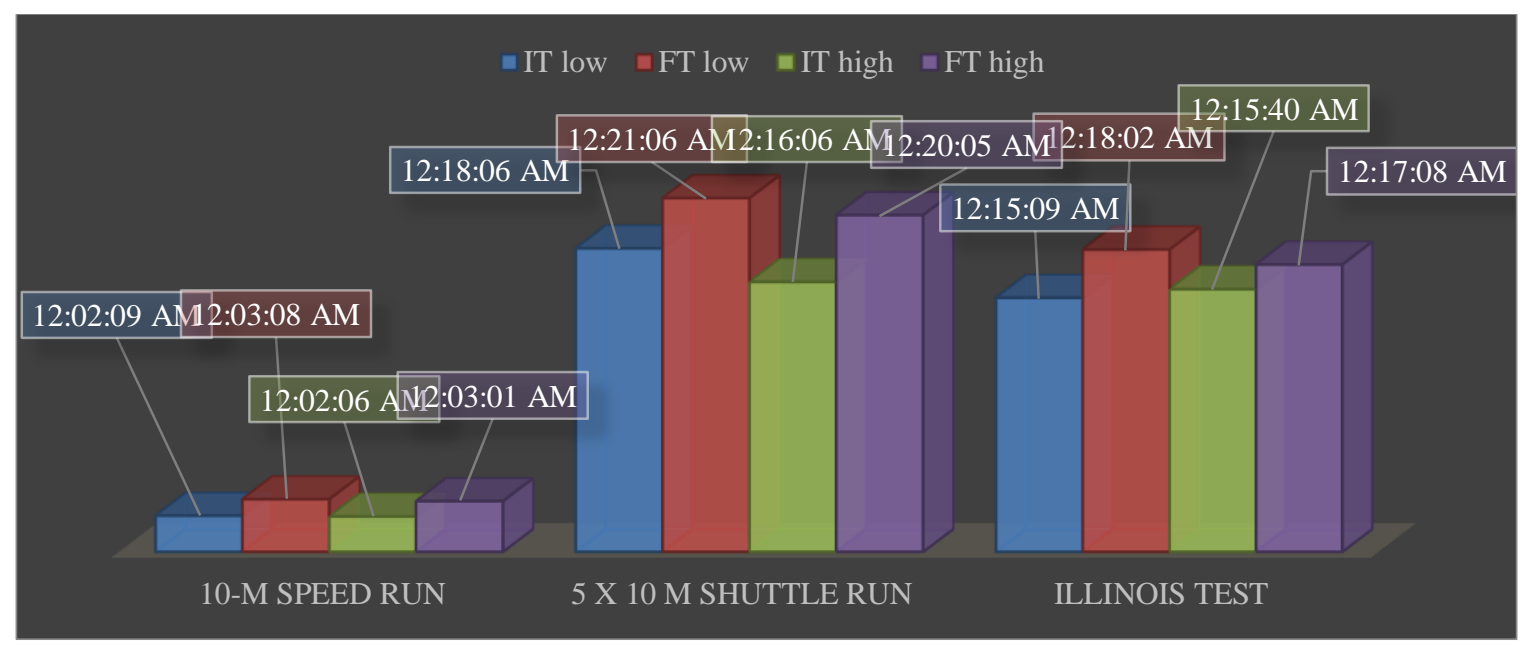

Figure 3. Physical qualities of motor skills

The test for motor skills related to the objectives of the training programme recorded important differences between the two times of testing, namely the initial test (IT) and final test (FT).

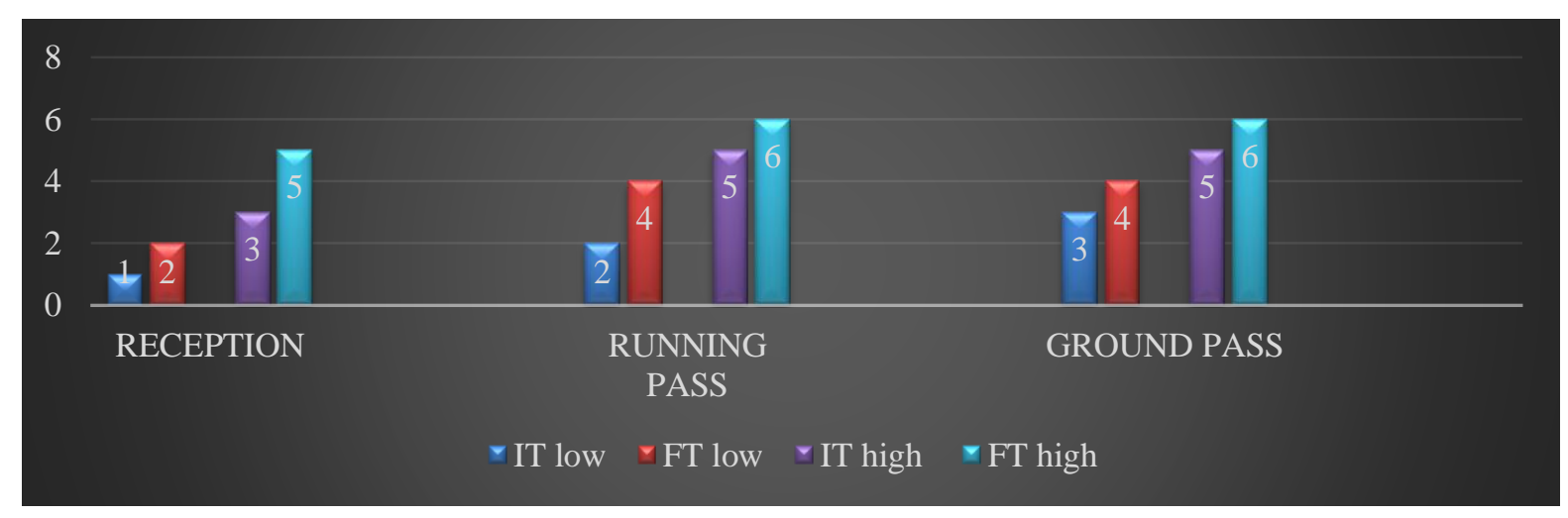

Figure 4. Results for motor skills in speed conditions

\section{Conclusion}

The collaboration with this team is 3 years old, besides the fact that young rugby players do not have a long history in the world of sports, especially in the game of rugby. In order to face this problem, a 3-week period was provided at the beginning of the special training period to initiate the basic movements, given that the tested rugby players had between 1 and 4 years of experience on the playing field. It should be noted that a third of them did not have any experience in this regard, meaning that the process of initiating or correcting movements needed a more detailed approach, unlike players coming from other sports, who had more developed motor skills.

At the same time, we managed to reduce the difference between the two categories of players, thus contributing to the homogeneity of the team and improving the individual and 
collective physical potential, while observing the timing of actions between the players of the rugby sevens team.

In the first weeks of the preparation programme, the emphasis was placed on the movement execution technique, either the players needed initiation or improvement, choosing a low- intensity execution and a high number of repetitions, and subsequently adding technical and tactical actions in the training process.

The improvement process did not take too long from the periods proposed for the development of physical potential, this allowing us to accelerate and increase the intensity of the training process, directing our attention to the relationship between reaction speed and execution speed in the technical and tactical actions of the players.

Through a concrete staging of the preparation programme, it has been demonstrated that the activity could be carried out at a more complex level. At the same time, rugby players manage to transmit the best image of the game of rugby sevens at junior level.

\section{References}

Abernethy, B. (1993). Searching for the minimal essential information for skilled perception and action. Psychological Research, 55(2), 131-138. https://doi.org/10.1007/bf00419644

Cârstea, G. (2000). Teoria și metodica educației fizice și sportului [Theory and methodology of physical education and sport]. București: AN-DA.

Cressey, E. M., West, C. A., Tiberio, D. P., Kraemer, W. J., \& Maresh, C. M. (2007). The effects of ten weeks of lower-body unstable surface training on markers of athletic performance. Journal of Strength and Conditioning Research, 21(2), 561-567. https://doi.org/10.1519/R-19845.1

Docherty, D., Wenger, H. A., \& Neary, P. (1988). Time-motion analysis related to the physiological demands of rugby. Journal of Human Movement Studies, 14, 269-277.

Duthie, G., Pyne, D., \& Hooper, S. (2003). Applied physiology and game analysis of rugby union. Sports Medicine, 33(13), 973-991. https://doi.org/10.2165/00007256-200333130-00003

Farrow, D., Young, W., \& Bruce, L. (2005). The development of a test of reactive agility for netball: A new methodology. Journal of Science and Medicine in Sport, 8(1), 52-60. https://doi.org/10.1016/s1440-2440(05)80024-6

Gabbett, T. J. (2005). Science of rugby league football: A review. Journal of Sports Sciences, 23(9), 961-976. https://doi.org/10.1080/02640410400023381

Gabbett, T. J. (2006). Skill-based conditioning games as an alternative to traditional conditioning for rugby league players. Journal of Strength and Conditioning Research, 20(2), 309-315. https://doi.org/10.1519/r-17655.1

Gabbett, T., Kelly, J., Ralph, S., \& Driscoll, D. (2009). Physiological and anthropometric characteristics of junior elite and sub-elite rugby league players, with special reference to starters and non-starters. Journal of Science and Medicine in Sport, 12(1), 215-222. https://doi.org/10.1016/j.jsams.2007.06.008

Galpin, J. A., Li, Y., Lohnes, C. A., \& Schilling, B. K. (2008). A 4-week choice foot speed and choice reaction training program improves agility in previously non-agility trained, but active men and women. Journal of Strength and Conditioning Research, 22(6), 19011907. https://doi.org/10.1519/jsc.0b013e3181887e3f

Green, B. S., Blake, C., \& Caulfield, B. M. (2011). A valid field test protocol of linear speed and agility in rugby union. Journal of Strength and Conditioning Research, 25(5), 12561262. https://doi.org/10.1519/jsc.0b013e3181d8598b 
Lloyd, R. S., \& Oliver J. L. (2012). The youth physical development model: A new approach to long-term athletic development. Strength \& Conditioning Journal, 34(3), 61-72. doi: 10.1519/SSC.0b013e31825760ea

Peterson, M. D., Alvar, B. A., \& Rhea, M. R. (2006). The contribution of maximal force production to explosive movement among young collegiate athletes. Journal of Strength and Conditioning Research, 20(4), 867-873. https://doi.org/10.1519/r-18695.1

Pichardo, A. W., Oliver, J. L., Harrison, C. B., Maulder, P. S., Lloyd, R. S., \& Kandoi, R. (2019). The influence of maturity offset, strength, and movement competency on motor skill performance in adolescent males. Sports, 7(7): 168. https://doi.org/10.3390/sports7070168

Sheppard, J. M., \& Young, W. B. (2006). Agility literature review: Classifications, training and testing. Journal of Sports Sciences, 24(9), 919-932. https://doi.org/10.1080/02640410500457109

Sheppard, J. M., Young, W. B., Doyle, T. L., Sheppard, T. A., \& Newton, R. U. (2006). An evaluation of a new test of reactive agility and its relationship to sprint speed and change of direction speed. Journal of Science and Medicine in Sport, 9(4), 342-349. https://doi.org/10.1016/j.jsams.2006.05.019

Taylor, J. M., Cunningham, L., Hood, P., Thorne, B., Irvin, G., \& Weston, M. (2019). The reliability of a modified 505 test and change-of-direction deficit time in elite youth football players. Science and Medicine in Football, 3(2), 157-162. https://doi.org/10.1080/24733938.2018.1526402

Ursanu, G. (2015). Optimizarea capacităţii motrice [Optimisation of motor ability]. Iași: UAIC.

Van Hooren, B., \& De Ste Croix, M. B. (2020). Sensitive periods to train general motor abilities in children and adolescents. Strength \& Conditioning Journal, 42(6), 7-14. https://doi.org/10.1519/SSC.0000000000000545

Young, W. B., James, R., \& Montgomery, I. (2002). Is muscle power related to running speed with changes of direction? Journal of Sports Medicine and Physical Fitness, 42, 282-288. https://pubmed.ncbi.nlm.nih.gov/12094116/ 